\title{
Characterisation of thermal crosstalk- induced wavelength shift in monolithic InP dual DFB lasers PIC
}

Lo, Mu-Chieh, Zhou, Zichuan, Pan, Shujie, Carpintero, Guillermo, Liu, Zhixin

Mu-Chieh Lo, Zichuan Zhou, Shujie Pan, Guillermo Carpintero, Zhixin Liu, "Characterisation of thermal crosstalk-induced wavelength shift in monolithic InP dual DFB lasers PIC," Proc. SPIE 11364, Integrated Photonics Platforms: Fundamental Research, Manufacturing and Applications, $113641 \mathrm{U}$ (4 May 2020); doi: 10.1117/12.2570618

SPIE. Event: SPIE Photonics Europe, 2020, Online Only 


\title{
Characterisation of thermal crosstalk-induced wavelength shift in a monolithic InP dual DFB lasers PIC
}

\author{
Mu-Chieh Lo ${ }^{\mathrm{a}}$, Zichuan Zhou ${ }^{\mathrm{a}}$, Shujie Pan ${ }^{\mathrm{a}}$, Guillermo Carpintero ${ }^{\mathrm{b}}$, and Zhixin Liu ${ }^{\mathrm{a}}$ \\ ${ }^{a}$ University College London, London, UK \\ bUniversidad Carlos III de Madrid, Leganés, Spain
}

\begin{abstract}
We present dual DFB lasers each integrated with one heater developed in a generic foundry platform. The thermal effects are experimentally investigated and exhibited a continuous wavelength difference tuning of 0-12.33 nm.
\end{abstract}

Keywords: Tunable lasers, Thermal effects, Photonic integrated circuits

\section{INTRODUCTION}

Dual continuous wave $(\mathrm{CW})$ lasers in InP-based C-band photonic integrated circuits (PIC) have become increasing promising, as they are capable to support the generation of highly advanced modulation format signals, ${ }^{1}$ millimeter wave $(\mathrm{mmW})$ and terahertz $(\mathrm{THz})$ carriers. ${ }^{2,3}$ To meet the regulated $\mathrm{mmW} / \mathrm{THz}$ frequency range and the channel grid given the tight constraints on the wavelength in optical networks, precise spectral tunability of compact devices is essential that is accomplished through injection current tuning and temperature tuning. It is worth mentioning that operation of laser or SOA leads to an additional heating in the gain section, as the thermo-optic coefficient for InP is about $2.5 \times 10^{-4}{ }^{\circ} \mathrm{C}^{-1}$ and thus thermal effects are inherently present. ${ }^{4}$

The thermal effects also give rise to unwanted thermal crosstalk, whereby a component is inevitably influenced by the temperature gradience of a neighbouring heating active component. ${ }^{5}$ Besides considering the thermal management approaches beyond the current standard macroscopic thermo-electric cooling (TEC) and conductive heat transfer, to keep a sufficient distance between components in PIC may significantly reduce the thermal crosstalk. ${ }^{6,7}$ However, it limits the PIC building block density and increases the development cost which conflicts with the concept of photonic integration and generic foundry approach. ${ }^{8}$

In this paper, we demonstrate a monolithically integrated dual DFB laser chip developed in an InP-based generic foundry platform. ${ }^{9}$ The DFB lasers are placed $400 \mu \mathrm{m}$ from each other in parallel and each one comes with an integrated heater electrode. The thermo-optic tuning capacity and the thermal crosstalk effects of DFB lasers on the DFB laser injection currents and heater currents are experimentally investigated. The dual peak wavelengths and their separation due to the thermal effects are analyzed to provide the thermal characteristics of the foundry technology as toolbox to all generic approach users in their design phase.

Further author information: (Send correspondence to Mu-Chieh Lo)

Mu-Chieh Lo: m.lo@ucl.ac.uk

Guillermo Carpintero: guiller@ing.uc3m.es

Zhixin Liu: zhixin.liu@ucl.ac.uk

Integrated Photonics Platforms: Fundamental Research, Manufacturing and Applications, edited by

Roel G. Baets, Peter O'Brien, Laurent Vivien, Proc. of SPIE Vol. 11364, 113641U

(C) 2020 SPIE · CCC code: $0277-786 X / 20 / \$ 21 \cdot$ doi: $10.1117 / 12.2570618$

Proc. of SPIE Vol. $11364113641 \mathrm{U}-1$ 


\section{DEVICE DESCRIPTION}
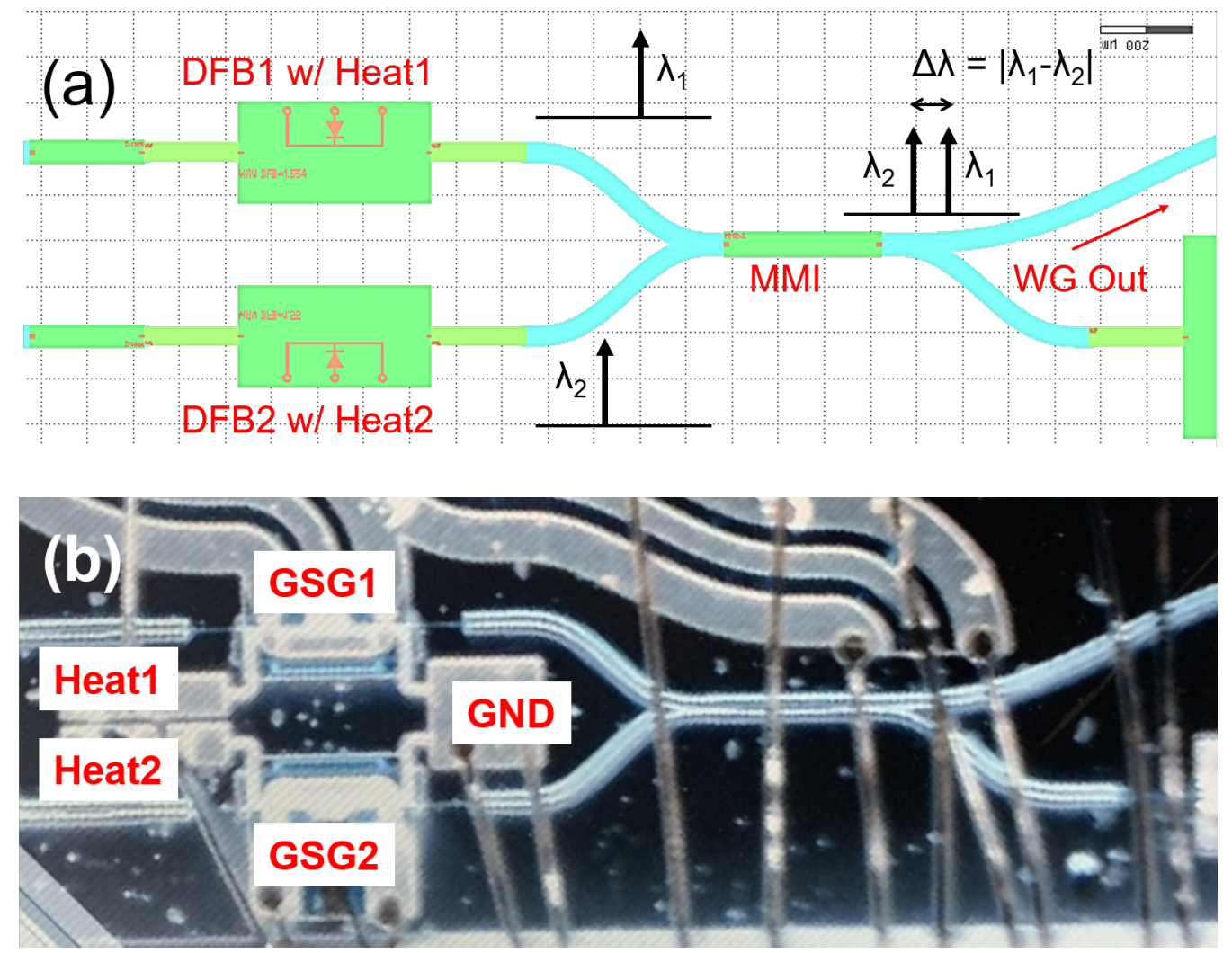

Figure 1. (a) PIC Mask layout. Blue: passive waveguides. Green: DFB laser, shallow-to-deep transition, and coupler building blocks. (b) PIC micro photo. Injection currents and heater currents are fed via GSG1,2 and Heat1,2 electrodes to DFB1 and DFB2, respectively.

The developed PIC mask layout and micro photo are shown in Fig. 1. Fig. 1(a) presents the two freely tunable DFB lasers with heaters are optically coupled through a MMI (multi-mode interference) coupler. Each DFB laser supports both injection current and thermal tuning mechanisms, via the injection current and heater current bonding pads (GSG and Heat, respectively) which are depicted in Fig. 1(b). After the MMI, the combined two wavelengths $\left(\lambda_{1}\right.$ and $\left.\lambda_{2}\right)$ are delivered to the upper waveguide out (WG Out) on cleaved facet, to which an external fiber is edge-coupled followed by measuring instruments for characterization. Under TEC control at $25^{\circ} \mathrm{C}$ the dependency of peak wavelengths $\lambda_{1}, \lambda_{2}$ and wavelength difference $\Delta \lambda=\left|\lambda_{1}-\lambda_{2}\right|$ on heater currents (Heat1 and Heat2) and injection currents (DFB1 and DFB2) are collected and analyzed. 


\section{CHARACTERIZATION RESULTS}
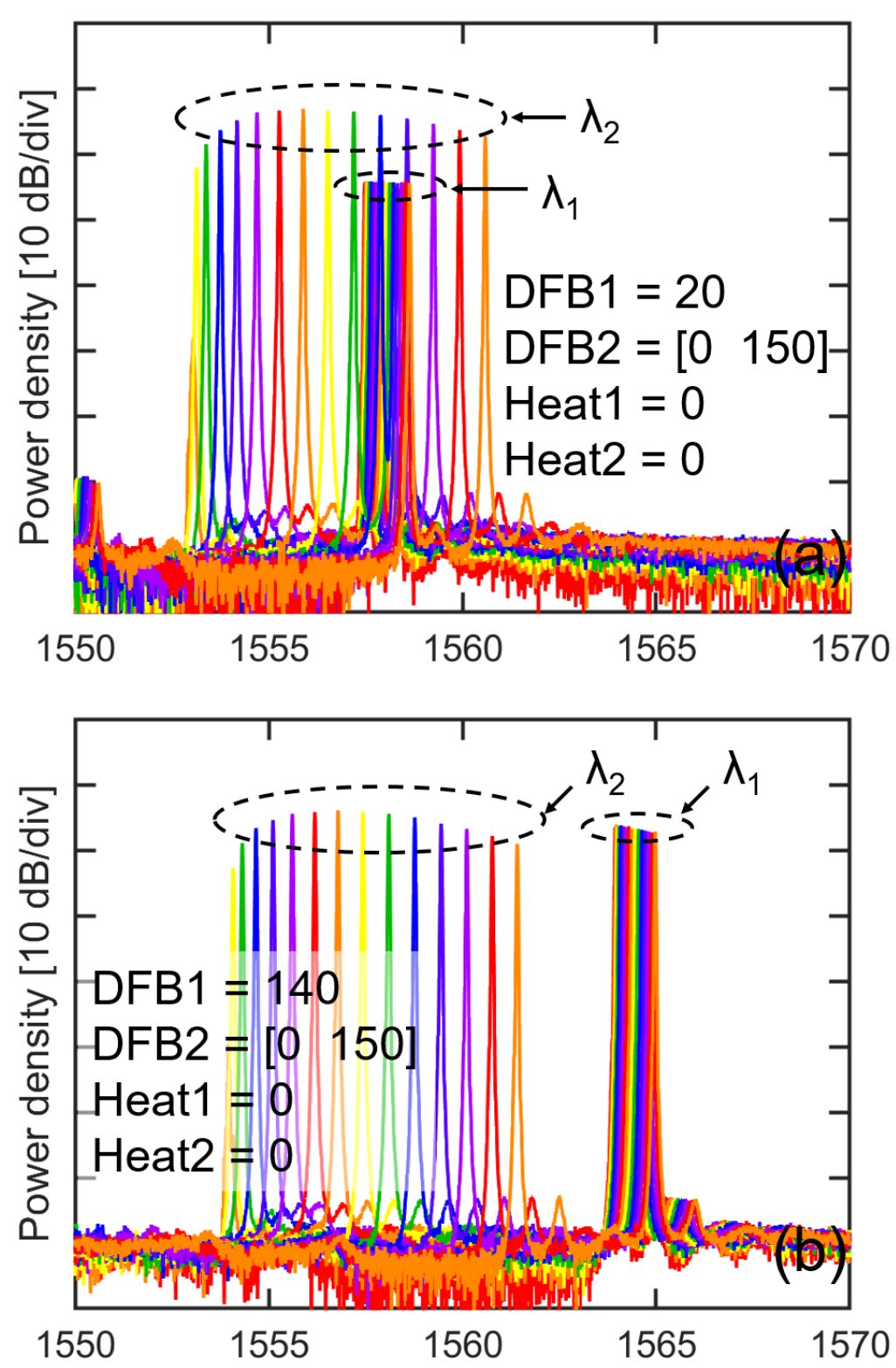

Figure 2. (a) Optical spectra for DFB1 fixed at $20 \mathrm{~mA}$ and DFB2 varied from 0 to $150 \mathrm{~mA}$. (b) Optical spectra for DFB1 fixed at $20 \mathrm{~mA}$ and $D F B 2$ varied from 0 to $150 \mathrm{~mA}$.

Fig. 2(a) and Fig. 2(b) present the optical spectrum evolution for DFB2 driven from 0 to $150 \mathrm{~mA}$ with DFB1 fixed at 20 and $140 \mathrm{~mA}$. Within this range of operation, the peak wavelength of DFB1 $\left(\lambda_{1}\right)$ is slightly increased $\approx 1 \mathrm{~nm}$ since it is influenced by the heat generated by DFB2 and the peak wavelength of DFB2 $\left(\lambda_{2}\right)$ shifts by $\approx 6 \mathrm{~nm}$. The group of $\lambda_{2}$ in Fig. 2(b) also red-shifts by $\approx 1 \mathrm{~nm}$ with respected to that in Fig. 2(a). 

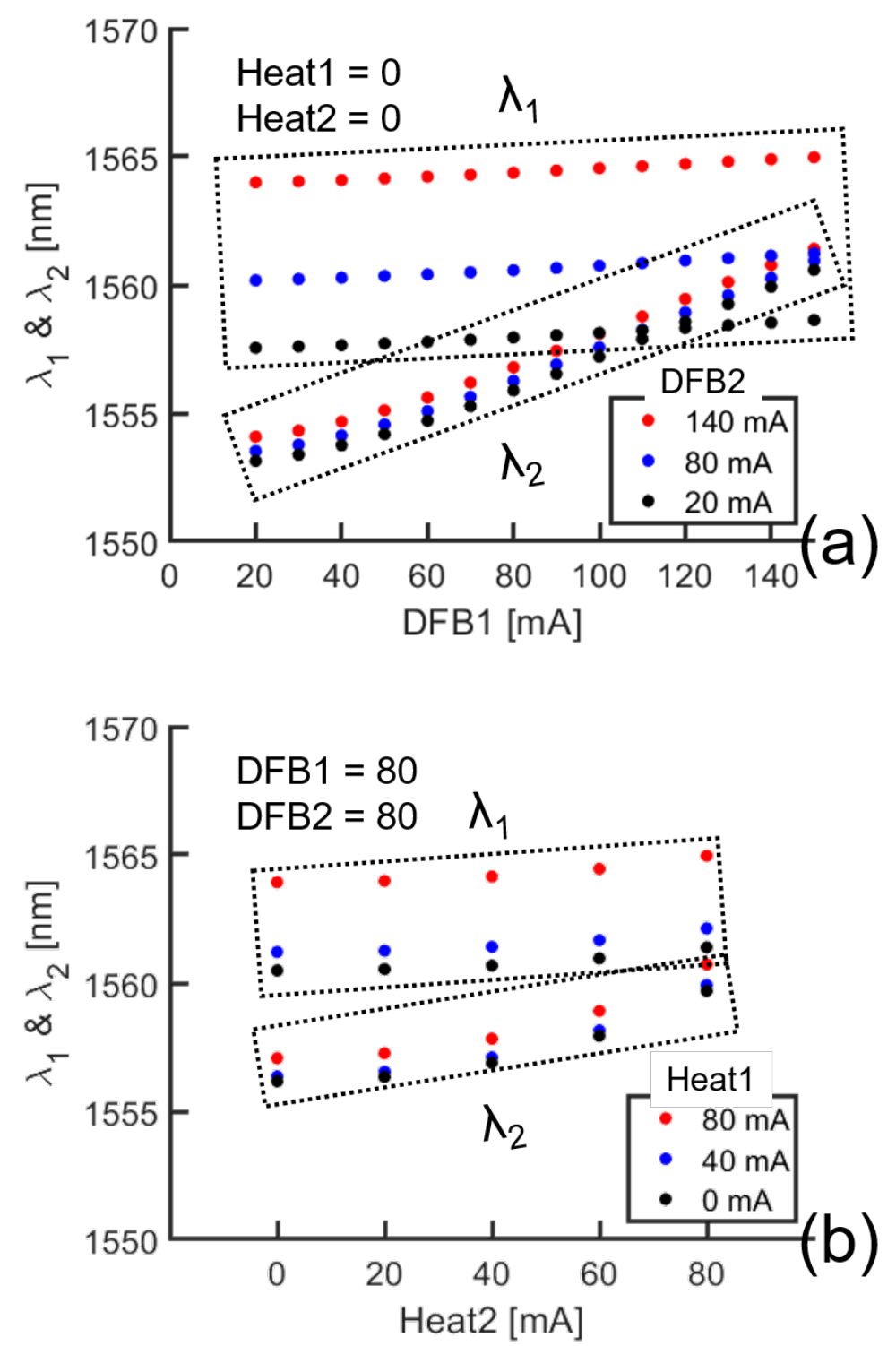

Figure 3. (a) Two peak wavelengths versus $D F B 1$ for $D F B 2=140,80$, and $20 \mathrm{~mA}$, and both heaters Heat1 and Heat2 off. (b) Two peak wavelengths against Heat2 for $D F B 1=D F B 2=80 \mathrm{~mA}$ and Heat1 switched off.

By extracting the peak wavelengths from the optical spectra, the dependence of the two peak wavelengths on DFB1 injection current is depicted in Fig. 3(a). For $D F B 1=20,80$, and $140 \mathrm{~mA}$, the three sets of dual peak wavelength data exhibit the monotonic upward trend of both $\lambda_{1}$ and $\lambda_{2}$ through the increasing $D F B 2$. $\lambda_{2}$ rises more rapidly than $\lambda_{1}$ while $D F B 2$ is consistently heating and the different rising rates may lead to wavelength crossing; For instance, when $D F B 1=20 \mathrm{~mA}$ and $D F B 2 \approx 110 \mathrm{~mA}$ the two lasers are in close spectral proximity, indicating the PIC can produce two equal wavelengths if it is properly biased. Similarly, Fig. 3(b) points out $\lambda_{1}$ and $\lambda_{2}$ are both thermally tuned by one single heater of the two, either Heat1 or Heat2. With a heater current of $80 \mathrm{~nm}$, the corresponding DFB laser red-shifts by $\approx 4 \mathrm{~nm}$ and the other DFB laser red-shifts by $\approx 1 \mathrm{~nm}$ due to the thermal crosstalk. 

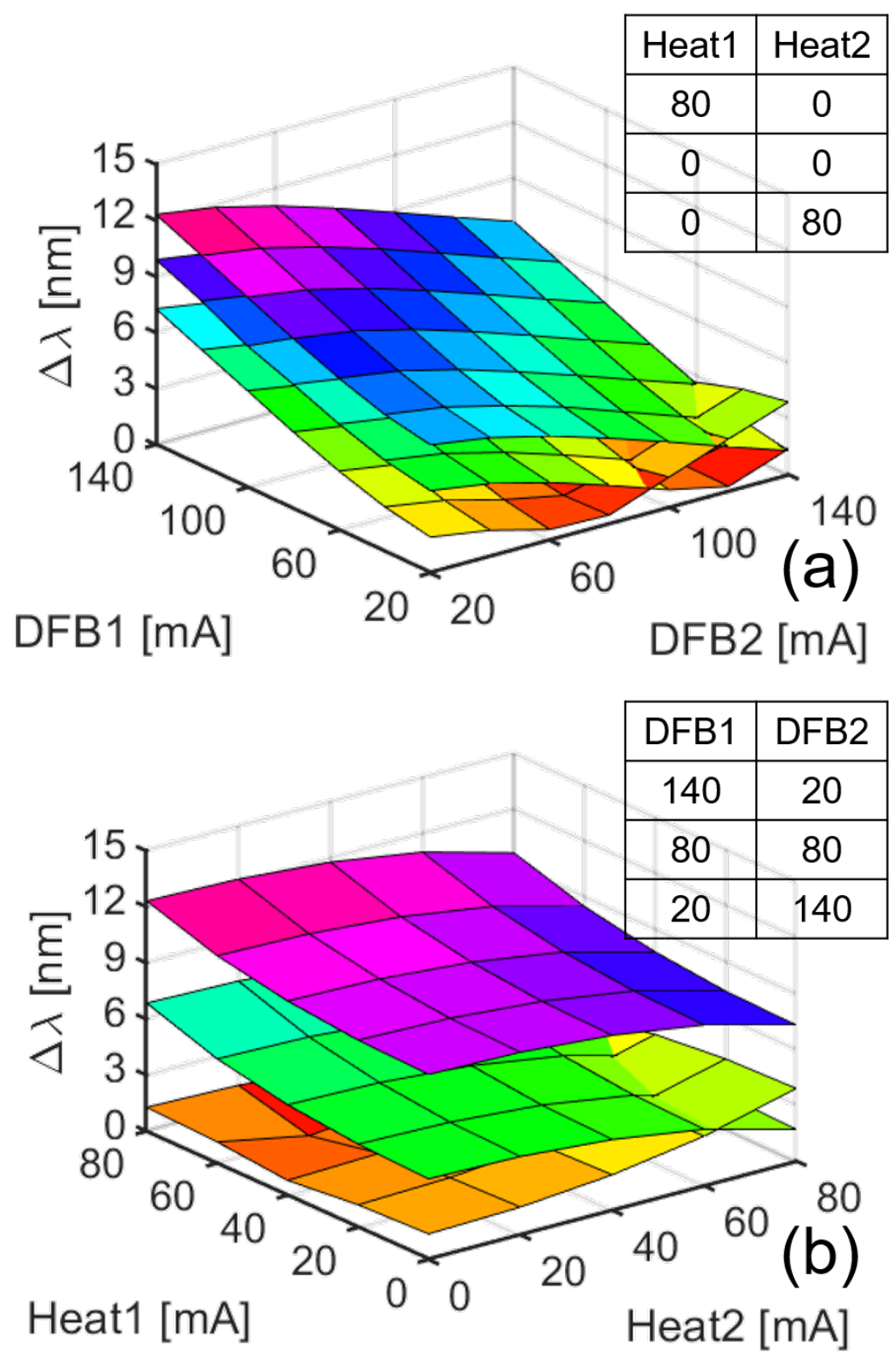

Figure 4. (a) 3D surface plots of wavelength difference against DFB1 and DFB2. (b) 3D surface plots of wavelength difference against Heat1 and Heat2.

The wavelength difference $(\Delta \lambda)$ is thus associated with the mutual influence, i.e., thermal crosstalk of the four variable factors: DFB1, DFB2, Heat1, and Heat2, which is visualized in Fig. 4(a) and Fig. 4(b). By sweeping the four variables, a four-dimension matrix is constructed and can be sliced to demonstrate the spectral information on a certain curved plane of interest. Each plane is composed of a range of linear or nonlinear wavelength dependence data as shown in Fig. 3(a) and Fig. 3(b). Furthermore, the widest $\Delta \lambda$ of $12.33 \mathrm{~nm}$ is reached when DFB1 and Heat1 are operated at the fairly large values (140 and $80 \mathrm{~mA})$ while the DFB2 is biased at $20 \mathrm{~mA}$ and the heater 2 is switched off $($ Heat2 $=0)$. On the other hand, the turning points of the planes reflect the $\Delta \lambda$ of $0 \mathrm{~nm}$, where the above-mentioned wavelength crossing occurs. 


\section{CONCLUSIONS}

In conclusion, the thermal effects and mutual influences of the PIC have been characterized. For each DFB laser, the tuning ranges of $6 \mathrm{~nm}$ and $4 \mathrm{~nm}$ for injection current tuning and thermal tuning and the $\approx 1 \mathrm{~nm}$ thermal crosstalk from the other laser as well as heater have been obtained. The PIC comprising dual DFB lasers with dual integrated microheaters features a wide tuning range of $0-12.33 \mathrm{~nm}$. Based on the $4 \mathrm{D}$ mapping, a pre-compensation scheme is now being evaluated to cancel the thermal crosstalk of the PIC ${ }^{10}$

\section{ACKNOWLEDGMENTS}

European Union's Horizon 2020 Marie Skłodowska-Curie grant agreement No.642355 and No.713694; Ministerio de Economía y Competitividad (MINECO) grant iTWIT (TEC2016-76997-C3-3-R) Engineering and Physical Sciences Research Council (EP/R035342/1, EP/R041792/1); Royal Society (PIF/R1/180001).

\section{REFERENCES}

[1] Liu, Z., Kakande, J., Kelly, B., O’Carroll, J., Phelan, R., Richardson, D. J., and Slavík, R., "Modulator-free quadrature amplitude modulation signal synthesis," Nature communications 5, 5911 (2014).

[2] Carpintero, G., Hisatake, S., de Felipe, D., Guzman, R., Nagatsuma, T., and Keil, N., "Wireless data transmission at terahertz carrier waves generated from a hybrid inp-polymer dual tunable dbr laser photonic integrated circuit," Scientific reports 8(1), 3018 (2018).

[3] Van Dijk, F., Kervella, G., Lamponi, M., Chtioui, M., Lelarge, F., Vinet, E., Robert, Y., Fice, M. J., Renaud, C. C., Jimenez, A., et al., "Integrated inp heterodyne millimeter wave transmitter," IEEE Photonics Technology Letters 26(10), 965-968 (2014).

[4] Della Corte, F. G., Cocorullo, G., Iodice, M., and Rendina, I., "Temperature dependence of the thermooptic coefficient of inp, gaas, and sic from room temperature to $600 \mathrm{k}$ at the wavelength of $1.5 \mu \mathrm{m}, "$ Applied Physics Letters 77(11), 1614-1616 (2000).

[5] Gilardi, G., Yao, W., Smit, M. K., and Wale, M. J., "Observation of dynamic extinction ratio and bit error rate degradation due to thermal effects in integrated modulators," Journal of Lightwave Technology 33(11), 2199-2205 (2015).

[6] Sato, K. and Murakami, M., "Experimental investigation of thermal crosstalk in a distributed feedback laser array," IEEE Photonics technology letters 3(6), 501-503 (1991).

[7] Mathews, I., Abdullaev, A., Lei, S., Enright, R., Wallace, M., and Donegan, J., "Reducing thermal crosstalk in ten-channel tunable slotted-laser arrays," Optics express 23(18), 23380-23393 (2015).

[8] Smit, M., Leijtens, X., Ambrosius, H., Bente, E., Van der Tol, J., Smalbrugge, B., De Vries, T., Geluk, E.-J., Bolk, J., Van Veldhoven, R., et al., "An introduction to inp-based generic integration technology," Semiconductor Science and Technology 29(8), 083001 (2014).

[9] Soares, F. M., Baier, M., Gaertner, T., Grote, N., Moehrle, M., Beckerwerth, T., Runge, P., and Schell, M., "Inp-based foundry pics for optical interconnects," Applied Sciences 9(8), 1588 (2019).

[10] Milanizadeh, M., Aguiar, D., Melloni, A., and Morichetti, F., "Canceling thermal cross-talk effects in photonic integrated circuits," Journal of Lightwave Technology 37(4), 1325-1332 (2019). 